\title{
Long-term survivors with immunotherapy in advanced NSCLC: is 'cure' within reach?
}

\author{
Sara Monteverdi ${ }^{1}$, Emanuele Vita ${ }^{2}$, Giulia Sartori ${ }^{1}$, Miriam Grazia Ferrara ${ }^{2}$, Ettore D'Argento ${ }^{3}$, \\ Giampaolo Tortora ${ }^{2,3}$, Michele Milella ${ }^{1}$, Emilio Bria ${ }^{2,3 *}$, Sara Pilotto ${ }^{1 *}$ \\ ${ }^{1}$ U.O.C. Oncology, University of Verona, Azienda Ospedaliera Universitaria Integrata, Verona, Italy; ${ }^{2}$ Medical Oncology, Università Cattolica del \\ Sacro Cuore, Roma, Italy; ${ }^{3}$ Comprehensive Cancer Center, Fondazione Policlinico Universitario Agostino Gemelli IRCCS, Roma, Italy \\ *These authors contributed equally for the last co-authorship. \\ Correspondence to: Emilio Bria. Professor, Medical Oncology, Fondazione Policlinico Universitario Agostino Gemelli IRCCS, Università Cattolica \\ Del Sacro Cuore, Largo Agostino Gemelli 8, 00168, Roma, Italy. Email: emilio.bria@unicatt.it. \\ Comment on: Garon EB, Hellmann MD, Rizvi NA, et al. Five-year overall survival for patients with advanced non-small-cell lung cancer treated with \\ pembrolizumab: results from the phase I KEYNOTE-001 study. J Clin Oncol 2019;37:2518-27.
}

Submitted Oct 13, 2019. Accepted for publication Dec 26, 2019.

doi: $10.21037 /$ tcr.2019.12.82

View this article at: http://dx.doi.org/10.21037/tcr.2019.12.82

Lung cancer represents the main cause of cancer-related death globally. In recent years, the introduction of immune checkpoint inhibitors (ICIs) have changed prognosis in a relevant proportion of non-small cell lung cancer (NSCLC) patients, especially in immunogenic tumors, such as the smoke-related ones.

Pembrolizumab is the only ICI approved as monotherapy in the first-line setting of non-oncogene addicted NSCLC harboring programmed death ligand-1 (PD-L1) expression on at least $50 \%$ of tumor cells. The recently published 5 -year outcomes of phase Ib KEYNOTE (KN)-001 trial is the longest efficacy and safety update for patients affected by NSCLC treated with immunotherapy (1). This study demonstrated the safety and efficacy with a prolonged overall survival (OS) benefit in NSCLC patients treated with pembrolizumab monotherapy (2). In $\mathrm{KN}-001$ trial, 550 patients with advanced NSCLC were treated with pembrolizumab monotherapy, with a schedule of $2 \mathrm{mg} / \mathrm{kg}$ every 3 weeks or $10 \mathrm{mg} / \mathrm{kg}$ every 2 or 3 weeks, and after a protocol amendment (April 2016) $200 \mathrm{mg}$ flat dose every 3 weeks. Patients were either treatment-naïve $(n=101)$ or pre-treated patients $(n=449)$. The primary study endpoint was objective response rate (ORR). Secondary endpoints were OS and duration of response (DoR). After 60.6 months of median follow-up, ORR was $41.6 \%$ [ $n=42 ; 95 \%$ confidence interval (CI): 31.9-51.8] in treatment-naive and $22.9 \%(\mathrm{n}=103 ; 95 \% \mathrm{CI}: 19.1-27.1)$ in previously- treated patients. In terms of secondary endpoints, median OS was 22.3 (95\% CI: 17.1-32.3) and 10.5 months (95\% CI: $8.6-13.2)$ with a 5 -year OS rate of $23.2 \%$ and $15.5 \%$ for treatment-naive and previously-treated patients, respectively. The greatest benefit was observed in patients treated frontline and with high PD-L1 expression [tumor proportion score (TPS) $\geq 50 \%$ ]. For PD-L1 $\geq 50 \%$ patients a 5 -year OS rate of $29.6 \%$ and a median OS of 35.4 months (95\% CI: 20.3-63.5) were reported. There was a positive correlation between PD-L1 expression and longer OS, higher ORR. A median DoR of 16.8 and 38.9 months were described for treatment-naive and pre-treated patients, respectively. In the subgroup of patients who received 2 or more years of pembrolizumab $(n=60)$, the estimated 5 -year OS was $78.6 \%$ in the first-line setting $(n=14)$ and $75.8 \%$ in the second and further-line group $(\mathrm{n}=46)$. Median DoR was 52.0 (range, 10.2-55.7) months for treatment-naive patients and not reached (range, 12.5-71.8) for previously-treated patients. Pembrolizumab monotherapy was overall welltolerated with grade 3 to 5 adverse events (AEs) occurring in $13 \%$ of patients $(n=69)$. The most common side effect was hypothyroidism (9\%) and the most serious was pneumonitis (5\%, of which $2 \%$ grade $3-5$ ).

According to the results of KN-001, the PD-L1 cut-off for selecting NSCLC patients eligible for ICI monotherapy in first-line was established at $\geq 50 \%$. In this setting, the phase III study $\mathrm{KN}-024$ validated the use of pembrolizumab 
monotherapy as first-line therapy in NSCLC highly expressing PD-L1 (3). In this trial, pembrolizumab demonstrated to improve the primary endpoint PFS, as well as OS and ORR, with a favorable toxicity profile compared with platinum-based chemotherapy. After a median follow-up of 44.4 months, median OS was 26.3 months with pembrolizumab and 14.2 months with chemotherapy [hazard ratio (HR): $0.65 ; 95 \%$ CI: $0.50-0.86 ; \mathrm{P}=0.001$ ]. Of interest, in the $\mathrm{KN}-024$ trial, OS benefit was observed although $64.9 \%$ of patients crossed over from chemotherapy to an anti-PD-(L)1 agent after disease progression (4). Similarly, the phase III KN-042 trial showed that pembrolizumab significantly prolonged OS over platinum-based chemotherapy as first-line therapy for non-oncogene addicted NSCLC expressing PD-L1 $\geq 1 \%$, although a greater magnitude of benefit was reported in the subpopulation with $\mathrm{PD}-\mathrm{L} 1 \geq 50 \%$ reporting a significant OS advantage (20.0 vs. 12.2 months, HR: 0.69; 95\% CI: $0.56-0.85 ; \mathrm{P}=0.0003)(5)$.

Based on a strong biological rationale, recent evidence from clinical trials in first-line have demonstrated that the combination of immunotherapy and chemotherapy (with or without antiangiogenic) or other immunotherapeutic agents provides a clinically meaningful benefit compared with standard-of-care in NSCLC patients, mainly irrespective of PD-L1 expression (6-15). Results of main clinical trials exploring immunotherapy in first-line treatment of NSCLC are listed in Table 1.

In the rapidly evolving landscape of lung cancer treatment, important questions have arisen mainly focusing on patient's selection for immunotherapy. As demonstrated by the KN-001 results, which are consistent with longterm follow-up of trials applying immunotherapy in pretreated NSCLC (16), a proportion of patients (around $15 \%$ ) is likely to achieve a long-term clinical benefit from immunotherapy alone. In this light, the crucial question is whether it is possible and how to identify these patients who may be spared from chemotherapy-immunotherapy combination (and its potential toxicity), without detrimentally impacting on their survival outcomes.

In terms of candidate predictors, to date no singularlyconsidered clinical factors allow the selection of those patients eligible to single agent immunotherapy instead of combination. In fact, in $\mathrm{KN}$ and IMpower trials the benefit deriving from immunotherapy (alone or in combination) was observed across all patients' subgroups. Histology did not significantly impact on immunotherapy efficacy, nor alone $(3-5)$ or in combination with chemotherapy $(6,7)$ or another immunotherapeutic agent (14). Among the populations of interest, the presence of liver metastases is associated with a poor prognosis and a decreased likelihood of response to pembrolizumab in patients affected by NSCLC (17). Immunotherapy alone, as well as in combination with chemotherapy has provided superior outcomes compared with chemotherapy, irrespective of liver metastases, although the worse overall prognosis $(18,19)$. In IMpower150 trial, including liver metastases as a prespecified stratification factor, the quadruple treatment with atezolizumab, carboplatin-paclitaxel and bevacizumab resulted in a 3.9-month difference in OS among patients with liver metastases, representing a $48 \%$ reduction in risk of disease progression or death (20). Although there is no clear evidence in randomized trials that patients with liver metastases should be treated differently than patients without, stratification based on presence of liver metastases is a reasonable perspective. Clinically meaningful populations, such as patients with ECOG Performance Status 2 or brain metastases, have been systematically excluded from clinical trials with immunotherapy therefore real-world data together with dedicated studies are fundamental in the investigation about effectiveness and safety of immunotherapy in a wider range of patients. Regarding elderly patients, pooled data from three randomized trials (KN-010, KN-024 and $\mathrm{KN}-042)$ showed improved OS in patients ages $\geq 75$ years affected by advanced PD-L1-positive NSCLC treated with pembrolizumab versus those treated with chemotherapy (HR: 0.76; 95\% CI: 0.56-1.02) together with a more favorable safety profile and with a greater benefit in patients with $\mathrm{PD}$ L1 $\geq 50 \%$ (HR: $0.41 ; 95 \%$ CI: 0.23-0.73) (21). In KN189 study, the median age enrolled was around 65, making uncertain the tolerability and efficacy of combination in this patients' subgroup. With the available data, to date no indication coming from immunotherapy studies, either a single agent or a combination, has validated age as a choice criteria. Although in elderly patients immunotherapy alone may represent a reasonable option, candidate immunosenescence biomarkers have recently emerged and in future may be considered in selecting elderly patients for the most appropriate therapy. Another crucial aspect that clinicians should consider during treatment selection is toxicity. Although, randomized clinical trials as well as a systematic review and network meta-analysis including $\mathrm{KN}$ 189 and KN-407 studies found no significant increment in incidence of AEs grade $\geq 3$ between pembrolizumab plus chemotherapy and chemotherapy alone (22), this 
Table 1 Results of main clinical trials exploring immunotherapy in first-line treatment of NSCLC

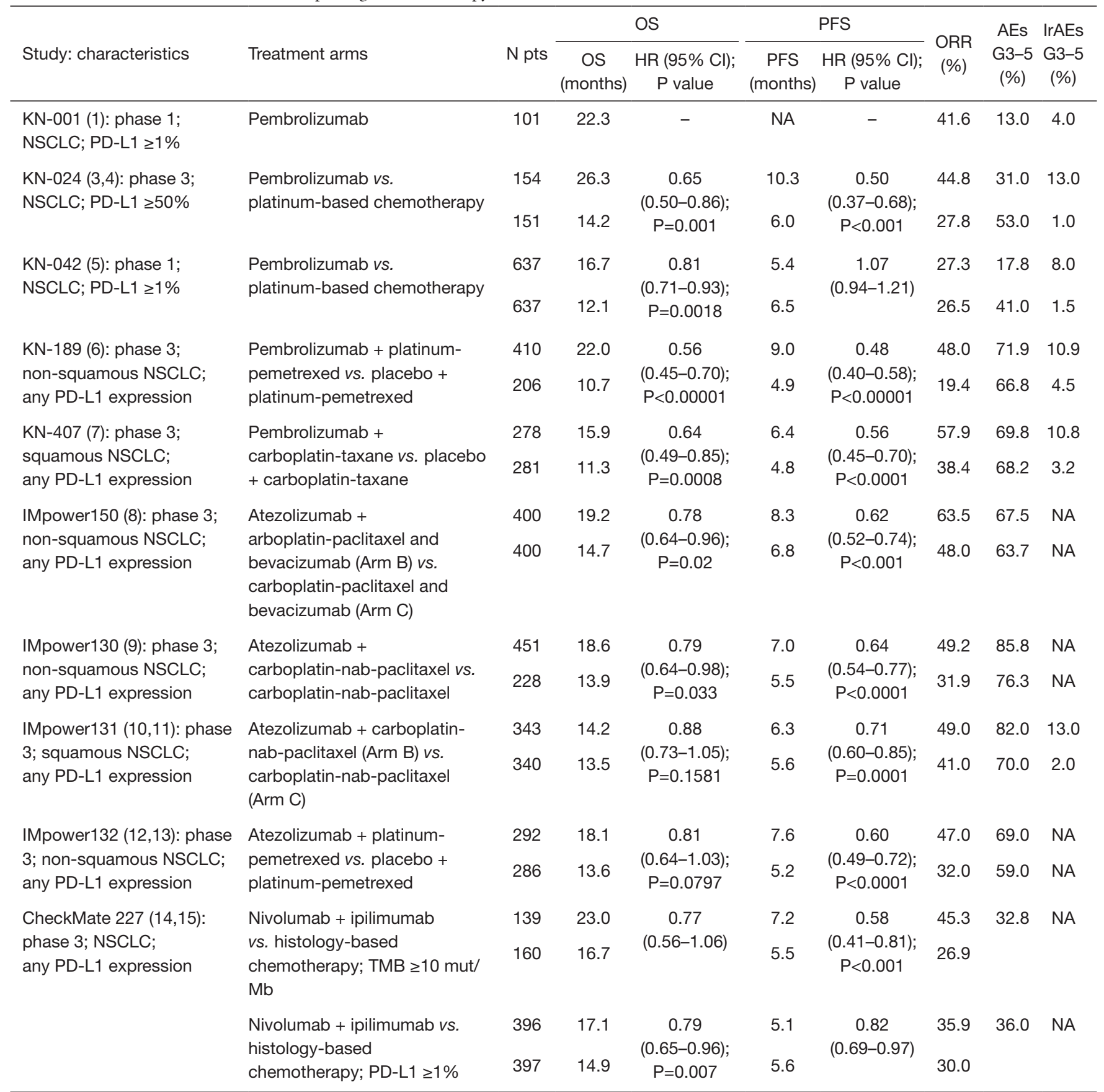

AEs of any cause are included in the table. KN-001: only data from treatment-naïve patients were reported (apart from toxicity); IMpower150: Arm B vs. Arm C data and results in the WT population are reported. IrAEs are only individually reported; IMpower130: IrAEs are only individually reported; IMpower131: Arm B vs. Arm C data are reported; IMpower132: IrAEs are only individually reported; CheckMate 227: the analysis of the dual primary endpoints are reported: PFS with nivolumab + ipilimumab vs. chemotherapy in patients with tumor mutational burden $\geq 10$ mut/Mb (OS from Press Release) and OS for nivolumab + ipilimumab vs. chemotherapy in pts with tumor PD-L1 $\geq 1 \%$ ). Treatment-related AEs are reported for all patients treated with nivolumab + ipilimumab or chemotherapy. IrAEs are only individually reported. OS, overall survival; PFS, progression free survival; ORR, overall response rate; AEs, adverse events; IrAEs, immune-related adverse events; N pts, number of patients; HR, hazard ratio; Cl, confidence interval; KN, KEYNOTE; NSCLC, non-small cell lung cancer; PD-L1, programmed death ligand-1; NA, not available; WT, wild type. 
percentage is relevantly higher when compared to monoimmunotherapy (Table 1). Moreover, patients included in trials of chemotherapy-immunotherapy combination were very selected with high screening failure rates, therefore the impact of toxicity in real life setting may be even higher than in clinical trials.

In terms of predictive bio-molecular factors, the association between tumoral PD-L1 expression and outcome is quite consistent among clinical trials with immunotherapy both alone and in combination. In strong positive PD-L1-expressing NSCLC, adding chemotherapy to pembrolizumab significantly decreased tumor size and delayed disease progression compared to pembrolizumab monotherapy $(\mathrm{P}<0.001)$ with a trend toward a quantitative interaction in OS $(\mathrm{P}=0.16)(23)$. When results of randomized clinical trials evaluating the combination of immunotherapy with chemotherapy (or chemotherapy-antiangiogenic agent) in first-line treatment of advanced NSCLC patients are analyzed with a meta-analytic approach, the combinatorial strategy provides a significant differential effect in terms of both OS and PFS, regardless of PD-L1 expression on tumor and/or immune cells (24). Considering the lack of an exclusive correlation with response/outcome, PD-L1 expression alone is not sufficient to explain the rates of long-term benefit described in patients affected by different cancer types, therefore other biomarkers are currently under investigation. Among them, tumoral mutational burden (TMB) has long been considered a candidate predictive biomarker for immunotherapy. Recent findings showed no significant association between TMB and efficacy of pembrolizumab-platinum-based chemotherapy, as well as nivolumab-ipilimumab, versus platinum-based chemotherapy alone, regardless of histology $(15,25)$. Based on this data, the scientific community agreed that TMB is not ready for being included into the therapeutic decisionmaking process.

Although the detection of a 'classic' oncogene-addiction (mainly EGFR mutations and $A L K$ translocations) has been recognized as a negative predictor for immunotherapy alone activity and efficacy, several genomic alterations are currently under investigation to define their potential prognostic and/or predictive impact. Among them, STK11, KEAP1 and ARID1A mutations are in the spotlight.

In conclusion, 5 years results of $\mathrm{KN}-001$ study have confirmed that immunotherapy alone has long term efficacy in a subgroup of NSCLC patients (similarly to previous findings in pre-treated NSCLC), but for the first time this prolonged benefit was extended to treatment-naïve patients, mainly strong PD-L1-expressors. In the rapidly changing landscape of lung cancer cure, where the combination of immunotherapy-chemotherapy recently revolutionized treatment possibilities, this finding strengthens the need to identify and validate innovative biomarkers in order to avoid overtreatment in those patients who may benefit from monotherapy alone. In light of the complexity of immune system-cancer interface, identifying a unique predictive biomarker is likely to be unrealistic and too simplifying. Thanks to the integration of different technologies, expertises and data, the recognition of crucial factors for immunotherapy response/resistance might lead to the real breakthrough point, allowing the accurate definition of the best therapeutical approach for each NSCLC patient.

\section{Acknowledgments}

Funding: Emilio Bria and Sara Pilotto are supported by the Italian Association for Cancer Research AIRC-IG 20583. Emilio Bria and Sara Pilotto were supported by the International Association for Lung Cancer (IASLC).

\section{Footnote}

Provenance and Peer Review: This article was commissioned and reviewed by the Section Editor Dr. Wei Xu (Division of Respiratory Disease, Department of Geriatrics, the First Affiliated Hospital of Nanjing Medical University, Nanjing, China).

Conflicts of Interest: The authors have completed the ICMJE uniform disclosure form (available at http://dx.doi. org/10.21037/tcr.2019.12.82). Michele Milella reports personal fees from Pfizer, EUSA Pharma and Astra Zeneca. Emilio Bria received honoraria or speakers' fee from MSD, Astra-Zeneca, Celgene, Pfizer, Helsinn, Eli-Lilly, BMS, Novartis and Roche. Sara Pilotto received honoraria or speakers' fee from Astra-Zeneca, Eli-Lilly, BMS, Boehringer Ingelheim, MSD, Roche and Istituto Gentili. The other authors have no conflicts of interest to declare.

Ethical Statement: The authors are accountable for all aspects of the work in ensuring that questions related to the accuracy or integrity of any part of the work are 
appropriately investigated and resolved.

Open Access Statement: This is an Open Access article distributed in accordance with the Creative Commons Attribution-NonCommercial-NoDerivs 4.0 International License (CC BY-NC-ND 4.0), which permits the noncommercial replication and distribution of the article with the strict proviso that no changes or edits are made and the original work is properly cited (including links to both the formal publication through the relevant DOI and the license). See: https://creativecommons.org/licenses/by-ncnd/4.0\%.

\section{References}

1. Garon EB, Hellmann MD, Rizvi NA, et al. Five-year overall survival for patients with advanced non-smallcell lung cancer treated with pembrolizumab: results from the phase I KEYNOTE-001 study. J Clin Oncol 2019;37:2518-27.

2. Garon EB, Rizvi NA, Hui R, et al. Pembrolizumab for the treatment of non-small-cell lung cancer. N Engl J Med 2015;372:2018-28.

3. Reck M, Rodríguez-Abreu D, Robinson AG, et al. Pembrolizumab versus chemotherapy for PD-L1positive non-small-cell lung cancer. N Engl J Med 2016;375:1823-33.

4. Reck M, Rodríguez-Abreu D, Robinson AG, et al. OA14. 01 KEYNOTE-024 3-year survival update: pembrolizumab vs platinum-based chemotherapy for advanced non-small-cell lung cancer. J Thorac Oncol 2019; 14:S243.

5. Mok TSK, Wu YL, Kudaba I, et al. Pembrolizumab versus chemotherapy for previously untreated, PD-L1expressing, locally advanced or metastatic non-small-cell lung cancer (KEYNOTE-042): a randomised, open-label, controlled, phase 3 trial. Lancet 2019;393:1819-30.

6. Gadgeel SM, Garassino MC, Esteban E, et al. KEYNOTE-189: Updated OS and progression after the next line of therapy (PFS2) with pembrolizumab (pembro) plus chemo with pemetrexed and platinum vs placebo plus chemo for metastatic nonsquamous NSCLC. J Clin Oncol 2019;37:abstr 9013.

7. Paz-Ares L, Luft A, Vicente D, et al. Pembrolizumab plus chemotherapy for squamous non-small-cell lung cancer. $\mathrm{N}$ Engl J Med 2018;379:2040-51.

8. Socinski MA, Jotte RM, Cappuzzo F, et al. Atezolizumab for first-line treatment of metastatic nonsquamous
NSCLC. N Engl J Med 2018;378:2288-301.

9. West H, McCleod M, Hussein M, et al. Atezolizumab in combination with carboplatin plus nab-paclitaxel chemotherapy compared with chemotherapy alone as firstline treatment for metastatic non-squamous non-smallcell lung cancer (IMpower130): a multicentre, randomised, open-label, phase 3 trial. Lancet Oncol 2019;20:924-37.

10. Jotte RM, Cappuzzo F, Vynnychenko I, et al. IMpower131: Primary PFS and safety analysis of a randomized phase III study of atezolizumab+ carboplatin+ paclitaxel or nab-paclitaxel vs carboplatin+ nab-paclitaxel as $1 \mathrm{~L}$ therapy in advanced squamous NSCLC. J Clin Oncol 2018;36:LBA9000.

11. Jotte R, Cappuzzo F, Vynnychenko I, et al. OA14. 02 IMpower131: final OS results of carboplatin+ nabpaclitaxel \pm atezolizumab in advanced squamous NSCLC. J Thorac Oncol 2019;14:S243-4.

12. Papadimitrakopoulou V, Cobo M, Bordoni R, et al. IMpower132: PFS and safety results with 1L atezolizumab+ carboplatin/cisplatin + pemetrexed in stage IV non-squamous NSCLC.J Thorac Oncol 2018;13:S332-3.

13. Barlesi F, Nishio M, Cobo M, et al. LBA54 IMpower132: Efficacy of atezolizumab (atezo)+ carboplatin (carbo)/ cisplatin (cis)+ pemetrexed (pem) as 1L treatment in key subgroups with stage IV non-squamous non-small cell lung cancer (NSCLC). Ann Oncol 2018;29:mdy424.066.

14. Hellmann MD, Ciuleanu TE, Pluzanski A, et al. Nivolumab plus ipilimumab in lung cancer with a high tumor mutational burden. N Engl J Med 2018;378:2093-104.

15. Hellmann MD, Paz-Ares L, Bernabe Caro R, et al. Nivolumab plus ipilimumab in advanced non-small-cell lung cancer. N Engl J Med 2019;381:2020-31.

16. Gettinger S, Borghaei H, Brahmer J, et al. OA14. 04 five-year outcomes from the randomized, phase 3 trials CheckMate 017/057: nivolumab vs docetaxel in previously treated NSCLC. J Thorac Oncol 2019;14:S244-5.

17. Tumeh PC, Hellmann MD, Hamid O, et al. Liver metastasis and treatment outcome with anti-PD-1 monoclonal antibody in patients with melanoma and NSCLC. Cancer Immunol Res 2017;5:417-24.

18. Garassino MC, Gadgeel S, Esteban E, et al. Abstract CT043: Outcomes among patients (pts) with metastatic nonsquamous NSCLC with liver metastases or brain metastases treated with pembrolizumab (pembro) plus pemetrexed-platinum: results from the KEYNOTE-189 study. Cancer Research 2019;79:CT043. 
19. Vokes EE, Ready N, Felip E, et al. Nivolumab versus docetaxel in previously treated advanced non-small-cell lung cancer (CheckMate 017 and CheckMate 057): 3-year update and outcomes in patients with liver metastases. Ann Oncol 2018;29:959-65.

20. Reck M, Mok TSK, Nishio M, et al. Atezolizumab plus bevacizumab and chemotherapy in non-small-cell lung cancer (IMpower150): key subgroup analyses of patients with EGFR mutations or baseline liver metastases in a randomised, open-label phase 3 trial. Lancet Respir Med 2019;7:387-401.

21. Nosaki K, Hosomi Y, Saka H, et al. 103O_PR Safety and efficacy of pembrolizumab (Pembro) monotherapy in elderly patients (Pts) with PD-L1-positive advanced NSCLC: Pooled analysis from KEYNOTE-010, -024, and-042. Ann Oncol 2019;30:mdz063.001.

22. Dafni U, Tsourti Z, Vervita K, et al. Immune checkpoint

Cite this article as: Monteverdi S, Vita E, Sartori G, Ferrara MG, D’Argento E, Tortora G, Milella M, Bria E, Pilotto S. Long-term survivors with immunotherapy in advanced NSCLC: is 'cure' within reach? Transl Cancer Res 2020;9(2):409-414. doi: 10.21037/tcr.2019.12.82 inhibitors, alone or in combination with chemotherapy, as first-line treatment for advanced non-small cell lung cancer. A systematic review and network meta-analysis. Lung Cancer 2019;134:127-40.

23. Pilotto S, Carbognin L, Rossi A, et al. Avoiding chemotherapy for advanced nononcogene addicted NSCLC overexpressing PD-L1: rule or option? Semin Oncol 2018;45:176-80.

24. Rossi A, Noia VD, Gkountakos A, et al. PD-L1 for selecting non-small-cell lung cancer patients for first-line immuno-chemotherapy combination: a systematic review and meta-analysis. Immunotherapy 2019;11:921-30.

25. Paz-Ares L, Langer CJ, Novello S, et al. LBA80 Pembrolizumab (pembro) plus platinum-based chemotherapy (chemo) for metastatic NSCLC: Tissue TMB (tTMB) and outcomes in KEYNOTE-021, 189, and 407. Ann Oncol 2019;30:mdz394.078. 